\title{
Diagnostic précoce de la résistance de cultivars de riz (Oryza sativa $L$ ) à la toxicité aluminique
}

\author{
Tang Van Hai 1, V Houben 2, C Nzok Mbouti 1, JE Dufey ${ }^{1 *}$ \\ 1 UCL-science du sol, place Croix du Sud 2/10, B1348 Louvain-la-Neuve, Belgique ; \\ 2 INRA-science du sol, 65, route de Saint-Brieuc, F34042 Rennes, France
}

(Reçu le 8 mars 1993 ; accepté le 23 août 1993)

\begin{abstract}
Résumé - Les rendements des cultures en sols très acides sont souvent limités par la hausse des concentrations en aluminium dans la solution du sol. II est dès lors souhaitable de sélectionner des espèces et variétés végétales résistant à la toxicité aluminique. À cet effet, il est intéressant de disposer de critères permettant un jugement précoce de l'effet de l'aluminium sur le rendement escompté sans devoir attendre la maturité de la plante. L'étude rapportée ici examine si le rendement en grain de 11 cultivars de riz cultivés sur solutions nutritives riches en aluminium peut être relié à des caractéristiques de la plante mesurables au stade végétatif. II apparaît que ni la production de biomasse, ni les teneurs minérales $(\mathrm{K}, \mathrm{Ca}, \mathrm{Mg}, \mathrm{P}, \mathrm{Al})$ dans les parties aériennes et dans les racines au stade végétatif ne permettent de discriminer des classes de cultivars résistants ou sensibles à l'aluminium établies sur la base du rendement en grain. En revanche, on observe que la capacité de tallage (nombre total de talles par plant) constitue un critère remarquable, simple et précoce de discrimination des classes de tolérance aluminique, le tallage maximum étant atteint 35 à $45 \mathrm{j}$ après le repiquage. En corollaire, cette recherche démontre qu'il est hasardeux, comme ce fut très souvent pratiqué dans des études antérieures, d'évaluer la toxicité aluminique sur la base d'expériences arrêtées au stade végétatif pour des espèces cultivées en vue de la production de grain.
\end{abstract}

riz / Oryza sativa L / aluminium / culture hydroponique / tallage / sélection

\begin{abstract}
Summary - Early diagnosis of aluminium toxicity to rice cultivars (Oryza sativa L). Crop yields in very acidic soils are often limited by increasing aluminium concentrations in the soil solution. It is therefore desirable to select plant species and varieties resistant to Al toxicity. For plants like cereals it is useful to identify criteria that make possible early diagnosis of Al resistance with respect to grain yield without waiting for maturity. In this study, we evaluate whether the grain yield of 11 rice cultivars grown on nutrient solution with high Al concentration can be related to plant characteristics at the vegetative stage. We found that neither biomass production nor the mineral concentrations ( $K$, $\mathrm{Ca}, \mathrm{Mg}, P, \mathrm{Al}$ ) in shoots and roots of 35-day-old plants can discriminate classes of Al-resistant and Al-sensitive cultivars with respect to grain yield. However we observed that the tillering capacity (total number of tillers per plant) was a remarkable characteristic for early assessment of the effect of Al on grain production, as maximum tillering occurs 3545 days after planting. This is quite a simple criterion for wide use in plant selection. As an important conclusion, we can assert that, though often done in many previous studies, it is hazardous to assess Al toxicity at the vegetable stage for plant species that are cultivated for grain production.
\end{abstract}

rice / Oryza sativa $L$ / aluminium / solution culture / tillering / selection

* Correspondance et tirés à part 


\section{INTRODUCTION}

Dans les sols acides, très largement répandus dans le monde (Van Wambeke, 1976), la croissance des végétaux est souvent limitée par des carences nutritionnelles et/ou par l'augmentation de la concentration en aluminium ionique dans la solution du sol. La tolérance à de fortes concentrations aluminiques dépend en premier lieu de l'espèce végétale, mais on observe également une grande diversité de sensibilité parmi les variétés ou cultivars d'une même espèce. La valorisation agronomique des sols acides peut donc être envisagée sous 2 aspects complémentaires: i) apports d'amendements et de fertilisants organiques et minéraux en vue d'améliorer les conditions nutritionnelles; ii) recherche d'espèces et de variétés végétales adaptées à ces milieux. Cette problématique a fait l'objet de plusieurs synthèses bibliographiques récentes (Fageria et al, 1988a ; Robson, 1989 ; Wright, 1989 ; Taylor, 1991).

La sélection de cultivars spécifiquement résistant à l'aluminium pose un problème méthodologique particulier. En effet, il est impossible, en conditions de plein champ, de faire varier la seule concentration aluminique dans la solution du sol. Étant donné les équilibres entre phases (par exemple Lindsay, 1979 ; Sposito, 1989), des modifications de concentrations en $\mathrm{Al}$ soluble et échangeable s'accompagnent obligatoirement de modifications de $\mathrm{pH}$ et de modifications de disponibilité des éléments majeurs et mineurs, rendant impossible toute interprétation non ambiguë de l'effet spécifique de l'aluminium. On comprend donc que la plupart des études sur la toxicité/résistance à l'aluminium aient été conduites sur solutions nutritives où l'on peut faire varier la concentration totale en Al indépendamment de la concentration des autres éléments, à condition de rester dans des zones de sous-saturation par rapport à différentes phases solides impliquant l'aluminium.

Le choix de critères de tolérance à l'aluminium est particulièrement délicat pour les céréales. En effet, le rendement en grain paraît être le seul choix objectif, puisque c'est le but de ces cultures. Mais des cultures sur solutions nutritives menées jusqu'à maturité représentent un travail long et fastidieux. II est donc utile de rechercher des critères plus précoces qui permettent un criblage variétal large et rapide.

La présente note rapporte une étude sur la résistance à l'aluminium de 11 cultivars de riz. Les résultats obtenus à maturité sont confrontés à des caractéristiques mesurables au stade végétatif. La pertinence de critères précoces fréquemment retenus dans la littérature, sans qu'il ne soit fait explicitement référence à la production de grain, est évaluée.

\section{MATÉRIEL ET MÉTHODES}

Les mesures de résistance à l'aluminium ont porté sur 11 cultivars de riz (Oryza sativa $L$ ) fréquemment utilisés en riziculture inondée dans le delta du Mékong au Viêt-Nam : IR 60A, IR 64, IRON 142, IRON 168, VN 86G, A 13H, OM 961, LUA MUA U17, LUA MUA 16B, KSB 21.

La solution nutritive de base avait la composition suivante: $\mathrm{KH}_{2} \mathrm{PO}_{4} 193 \mu \mathrm{M}, \mathrm{KCl} 229.5 \mu \mathrm{M}, \mathrm{CaCl}_{2} 250$ $\mu \mathrm{M}, \mathrm{MgSO}_{4} 411 \mu \mathrm{M}, \mathrm{NH}_{4} \mathrm{NO}_{3} 714 \mu \mathrm{M}$, FeEDTA 18 $\mu \mathrm{M}, \mathrm{Al}_{2}\left(\mathrm{SO}_{4}\right)_{3} 37 \mu \mathrm{M}+$ oligo-éléments, soit, en mg• $\mathrm{L}^{-1}: \mathrm{P} 6, \mathrm{~K} 9, \mathrm{Ca} 10, \mathrm{Mg} 10, \mathrm{~N}-\mathrm{NH}_{4} 10, \mathrm{~N}-\mathrm{NO}_{3} 10, \mathrm{Fe}$ 1, Al 2. Deux autres solutions contenant 10 et $20 \mathrm{mg}$ $\mathrm{Al} \cdot \mathrm{L}^{-1}$ ont été préparées par addition de $\mathrm{Al}_{2}\left(\mathrm{SO}_{4}\right)_{3}$ à la solution de base. Le choix d'une concentration de base de $2 \mathrm{mg} \mathrm{Al} \cdot \mathrm{L}^{-1}$ est justifié par des recherches antérieures (Tang Van Hai et al, 1989) qui ont montré que de faibles concentrations en Al, de l'ordre de 1 à 3 $\mathrm{mg} \cdot \mathrm{L}^{-1}$, induisent une stimulation des processus métaboliques.

Les cultures ont été menées en chambre climatisée dans les conditions suivantes : photopériode $12 \mathrm{~h} / \mathrm{j}$ ( \pm 15 watts $\cdot \mathrm{m}^{-2}$ ) et $12 \mathrm{~h} /$ nuit, température $30^{\circ} \mathrm{C}$ le j et $25^{\circ} \mathrm{C}$ la nuit, humidité relative $80 \%$ le j et $90 \%$ la nuit.

Après germination et préculture sur les 3 types de solutions nutritives pendant 15 j, les plantules de chaque variété ont été repiquées en 2 lots.

- Lot 1 : 3 x 15 plantules homogènes de chaque variété dans 9 bacs de 20 I ( 3 solutions $\times 3$ répétitions), renouvellement hebdomadaire des solutions, compensation quotidienne de l'évapotranspiration par addition d'eau déminéralisée, récolte à $35 \mathrm{j}$.

- Lot 2 : $2 \times 5$ plantules de chaque variété repiquées sur pots individuels de $1,4 \mathrm{l}$, renouvellement des solutions nutritives tous les $2 \mathrm{j}$, récolte à maturité.

Le poids sec des racines et des parties aériennes des plantes à $35 \mathrm{j}$ a été mesuré après séchage à $80^{\circ} \mathrm{C}$. Après calcination à $450^{\circ} \mathrm{C}$, traitement des cendres par un mélange d'acides concentrés $\left(\mathrm{H}_{2} \mathrm{SO}_{4}\right.$ $95 \%, \mathrm{HNO}_{3} 65 \%, \mathrm{HClO}_{4} 65 \%$ en proportions volumiques identiques), et dilution à l'eau déminéralisée, les teneurs en éléments minéraux ont été mesurées sur la solution ainsi obtenue filtrée. L'Al a été dosé par la méthode de l'aluminon (Barnhisel et Bertsch, 1982), les concentrations en $\mathrm{Ca}, \mathrm{Mg}$ et $\mathrm{K}$ ont été déterminées par spectrophotométrie d'absorption atomique, et les teneurs en $P$ par colorimétrie après complexation au molybdophosphate (Olsen et Sommers, 1982).

Sur les plantes menées jusqu'à maturité, on a déterminé le nombre total de talles, le nombre de talles 
fertiles, le nombre de panicules, le nombre de grains pleins par panicule, le poids de grain par plant et le poids de 1000 grains.

\section{RÉSULTATS ET DISCUSSION}

Les rendements moyens en grain plein obtenus pour les 11 variétés de riz ont été de 21,1 ( $\sigma$ $10,3), 3,06(\sigma 3,30)$ et $0,29(\sigma 0,47) \mathrm{g} / 10$ plants pour les solutions contenant 2,10 et $30 \mathrm{mg} \mathrm{Al} \mathrm{L}^{-1}$ respectivement. L'effet dépressif de l'aluminium est donc extrêmement marqué dans la gamme de concentrations étudiée. Les coefficients de variation entre cultivars sont très élevés, ce qui traduit des comportements très diversifiés et donc un échantillonnage de variétés intéressant. $\mathrm{Vu}$ la production négligeable à $20 \mathrm{mg} \mathrm{Al} \cdot \mathrm{L}^{-1}$, ces données ne seront pas considérées dans l'analyse détaillée des résultats. Il faut noter que de nombreuses études menées au stade végétatif explorent des gammes de concentration en $\mathrm{Al}$ nettement plus étendues (Fageria et al, 1988b; Tang Van Hai et al, 1989). La référence à la production de grain paraît donc indispensable pour fixer les domaines de concentration en Al intéressants pour un criblage de tolérance.

La figure 1 permet de classer les cultivars selon leur production à 2 et $10 \mathrm{mg} \mathrm{Al} \cdot \mathrm{L}^{-1}$. Parmi les cultivars les plus productifs à $2 \mathrm{mg} \mathrm{Al} \cdot \mathrm{L}^{-1}, 2$ cultivars se distinguent par leur bonne tolérance à l'aluminium (IR 64 et VN 86G) et 3 autres par leur sensibilité (IR 60A, LUA MUA 16B et KSB 21).

Parmi les cultivars de productivité moyenne à $2 \mathrm{mg} \mathrm{Al} \cdot \mathrm{L}^{-1}, 2$ apparaissent bien tolérants à l'Al, A $13 \mathrm{H}$ et surtout $\mathrm{OM}$ 96I. Les autres cultivars (IRON 168, IRON 268, LUA MUA U17 et IRON

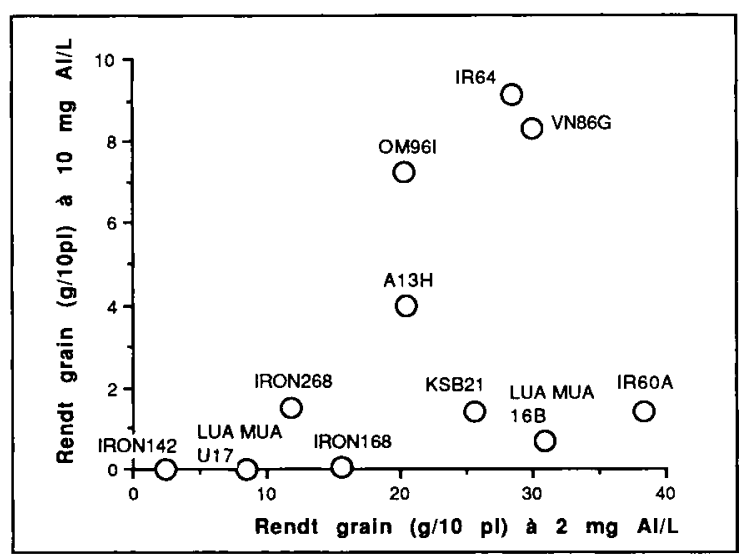

Fig 1. Production de grain fertile par les 11 cultivars de riz à 2 et $10 \mathrm{mg} \mathrm{Al} \cdot \mathrm{L}^{-1}$
142), de plus faible productivité, à $2 \mathrm{mg} \mathrm{Al} \cdot \mathrm{L}^{-1}$, se sont avérés très sensibles à l'aluminium.

Avant de rechercher des critères précoces s'accordant au mieux avec le classement de la figure 1 , il est utile d'examiner comment les caractéristiques mesurées à 35 j sont globalement affectées par f'aluminium en solution. Le tableau I compare les moyennes de ces caractéristiques pour les 11 cultivars à 2 et $10 \mathrm{mg} \mathrm{Al} \cdot \mathrm{L}^{-1}$.

Le poids moyen des parties aériennes n'est pas affecté par l'aluminium, alors que ce paramètre a souvent été retenu pour juger de la toxicité aluminique (Jan et Petterson, 1989 ; Fageria et al, 1988b), de préférence aux caractéristiques racinaires pourtant sensibles aux variations de concentration en $\mathrm{Al}$ à partir de seuils plus faibles. Le poids moyen de racine est, dans ce cas présent, réduit de près de moitié lorsque la concentration en Al passe de 2 à $10 \mathrm{mg} \cdot \mathrm{L}^{-1}$.

Tableau I. Caractéristiques des 11 cultivars de riz mesurables avant maturité. Comparaison des moyennes à 2 et $10 \mathrm{mg} \mathrm{Al} \cdot \mathrm{L}^{-1}$.

$2 \mathrm{mg} \mathrm{Al \cdot LL^{-1 }} 10 \mathrm{mg} \mathrm{Al \cdot LL^{-1 }}$ Signific $^{a}$

$\begin{array}{lccc}\text { Poids sec à } & 35 j(g / 15 p l) & & \\ \text { Feuilles } & 1,31(24)^{\mathrm{b}} & 1,36(18) & \text { NS } \\ \text { Racines } & 0,47(40) & 0,26(27) & \star \star\end{array}$

Teneurs minérales à $35 \mathrm{j}(\mathrm{mg} / \mathrm{g}$ MS)

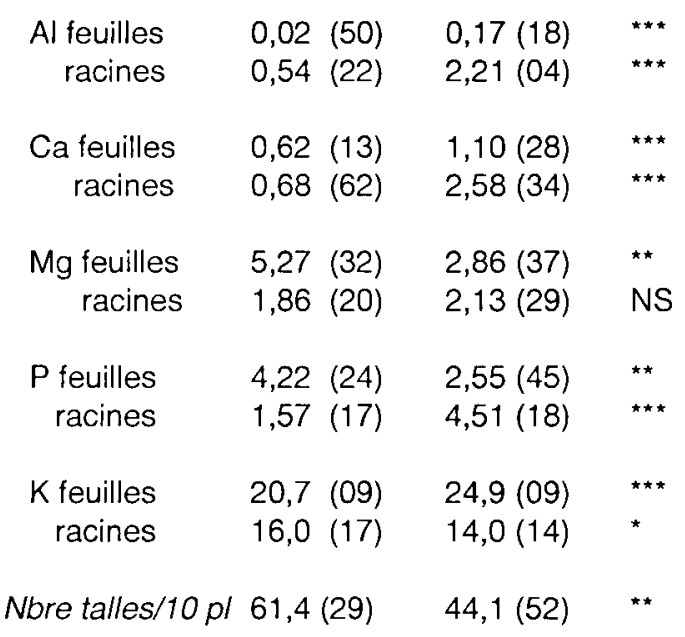

a Niveaux de signification des différences entre moyennes: NS : non significatif, " significatif $(\alpha 0,05),{ }^{*}$ hautement significatif $(\alpha 0,01),{ }^{* * *}$ très hautement significatif $(\alpha 0,001) ; b$ entre parenthèses : coefficient de variation (\%) entre les 11 cultivars. 
Les concentrations en éléments minéraux dans les feuilles et les racines sont très affectées par l'augmentation de la concentration en $\mathrm{Al}$ dans le milieu nutritif. Les racines s'enrichissent en aluminium, mais la translocation vers les parties aériennes reste limitée, du moins en valeur absolue. Le calcium subit une augmentation de teneur parallèle à celle de l'aluminium dans les racines.

La translocation du magnésium et du phosphore des racines vers les parties aériennes est freinée en présence d'aluminium dans le milieu. II s'agit là de l'effet le plus dommageable de l'aluminium du point de vue nutritionnel, et on a montré que l'augmentation des concentrations en $\mathrm{Mg}$ et $P$ dans le milieu nutritif est susceptible d'atténuer l'effet dépressif de l'aluminium (Tang Van Hai et al, 1989). D'un autre côté, la présence d'aluminium augmente fortement la concentration en $P$ dans les racines. Cela dénote sans doute un blocage du phosphore dans l'apoplasme par formation de complexes aluminophosphatés liés aux parois cellulaires (Taylor, 1991).

On note enfin que les concentrations moyennes en $\mathrm{K}$ sont, en valeur absolue, significativement affectées par l'aluminium, mais les différences relatives restent faibles. Le potassium est un élément ionique peu réactionnel (peu complexable, peu précipitable) dont le prélèvement actif est réglé notamment par l'activité de I'ATPase membranaire et l'extrusion de protons qui en résulte. Des résultats en sens divers sont rapportés dans la littérature quant à l'effet de l'aluminium sur ces processus (Kinraide, 1988) ; ce thème mérite assurément d'être approfondi.

Le nombre de talles par plant est une caractéristique qui peut être évaluée avant maturité pour autant que l'on ne doive pas distinguer talles fertiles et stériles. La phase de tallage débute 15-20 j après le repiquage et le maximum de talles est atteint après 35-45 j, la maturité intervenant après $90-130 \mathrm{j}$ chez la plupart des variétés cultivées actuellement. Le tableau I montre que le nombre moyen de talles est fortement réduit par la présence d'aluminium, mais les écarts entre variétés sont très importants (coefficient de variation de plus de $50 \%$ à $10 \mathrm{mg}$ Al. $\left.L^{-1}\right)$.

L'ensemble des données rassemblées au tableau I montre que l'on dispose de variables très sensibles à l'aluminium. L'examen des variations de ces paramètres selon les cultivars devrait pouvoir dégager des critères de plus ou moins grande sensibilité à l'aluminium.

Une analyse statistique par la méthode des composantes principales (Philippeau, 1986) a d'abord été effectuée pour la concentration en $\mathrm{Al}$ de $2 \mathrm{mg} \cdot \mathrm{L}^{-1}$. La question était de savoir si la variabilité de la production de grain (introduite comme variable supplémentaire) sans stress aluminique peut être liée à la variabilité générale des 11 cultivars définie par les caractéristiques mesurées avant maturité, et, dans l'affirmative, de vérifier si cette relation est maintenue sous stress aluminique. Les pourcentages de la variation totale expliqués par les 3 premiers axes sont de $39,4,14,3$ et $13,4 \%$. La partie supérieure de la figure 2 montre les cercles de corrélations pour les axes 1 et 2, et les axes 1 et 3 . II apparaît qu'à $2 \mathrm{mg} \mathrm{Al} \cdot \mathrm{L}^{-1}$ le rendement en grain (vecteur très court, presque perpendiculaire à l'axe principal) est très mal expliqué par toutes ces variations, les corrélations du rendement avec les axes 1,2 et 3 étant respectivement de $-0,06$, $-0,33$ et $-0,25$. On peut donc penser qu'en absence de stress aluminique les différences génotypiques entre cultivars s'expriment différemment

\section{Al $2 \mathrm{mg} / \mathrm{L}$}
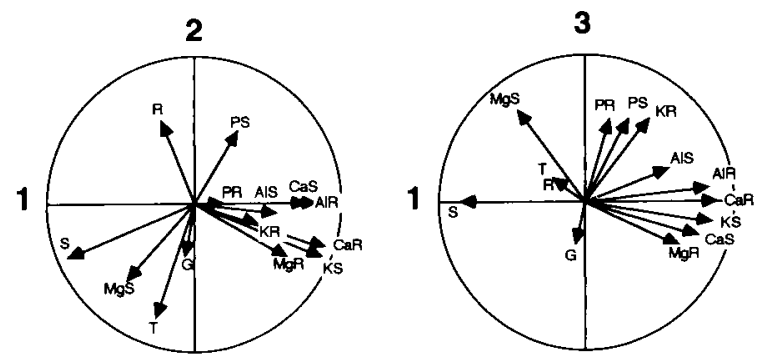

\section{Al $10 \mathrm{mg} /$}
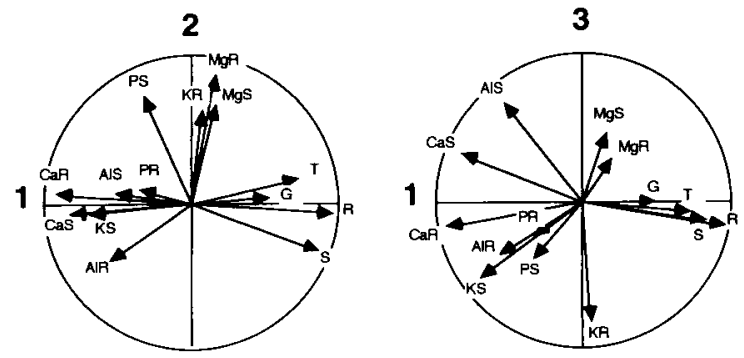

Fig 2. Cercles de corrélations entre les propriétés des 11 cultivars de riz obtenus par analyse en composantes principales. Signification des symboles : $\mathrm{S}=$ poids $\mathrm{sec}$ des parties aériennes à $35 \mathrm{j} ; \mathrm{R}=$ poids sec des racines à $35 \mathrm{j}$; AIS, $\mathrm{CaS}, \mathrm{MgS}, \mathrm{KS}, \mathrm{PS}=$ concentrations en $\mathrm{Al}, \mathrm{Ca}, \mathrm{Mg}, \mathrm{K}, \mathrm{P}$ dans les parties aériennes à $35 \mathrm{j}$; AIR, $\mathrm{CaR}, \mathrm{MgR}, \mathrm{KR}, \mathrm{PR}=$ concentrations en $\mathrm{Al}, \mathrm{Ca}, \mathrm{Mg}, \mathrm{K}, \mathrm{P}$ dans les racines à $35 \mathrm{j} ; \mathrm{T}$ $=$ nombre total de talles; $G=$ rendement en grain. 
sur le rendement en grain et sur les caractéristiques simples rapportées au tableau 1.

Une analyse semblable a été effectuée sur les données à $10 \mathrm{mg} \mathrm{Al} \cdot \mathrm{L}^{-1}$. Les pourcentages de variation expliqués par les 3 premiers axes sont de $39,3,20,1$ et $15,6 \%$, soit au total $70,5 \%$. La partie inférieure de la figure 3 présente les cercles de corrélations pour les axes 1 et 2 , et les axes 1 et 3 . On remarque que le vecteur de rendement en grain, introduit comme variable supplémentaire dans l'analyse, est très proche de l'axe principal avec lequel il présente une corrélation de 0,51 , alors que la corrélation avec les axes 2 et 3 est négligeable $(0,18$ et 0,01$)$. La variabilité du rendement en grain sous stress aluminique est donc corrélée à la variabilité des caractéristiques qui contribuent le plus à la définition de l'axe principal. Les coefficients de corrélation les plus significatifs entre la production du grain et les autres propriétés sont obtenus avec le poids des parties aériennes $(r=0,38)$, la concentration en Ca dans les feuilles $(r=0,39)$, le poids de racines $(r=0,47)$, la concentration en $\mathrm{Ca}$ dans les racines $\left(r=-0,60^{*}\right)$, et le nombre total de talles $\left(r=0,91^{\star \star *}\right)$.

La figure 3 présente la relation entre le rendement et le nombre total de talles. On y relève manifestement 2 parties : un domaine de nombre de talles pour lequel le rendement en grain est négligeable, et un domaine situé au-delà d'un seuil d'environ 5 talles par plant pour lequel la production en grain augmente fortement avec la capacité de tallage.

Selon les résultats présentés ici, si on souhaite se limiter à un critère simple de tolérance à l'aluminium mesurable avant maturité, la capacité maximum de tallage, observable $35-45 \mathrm{j}$ après le repiquage, présente un intérêt évident et mérite des études plus systématiques. La littérature indi-

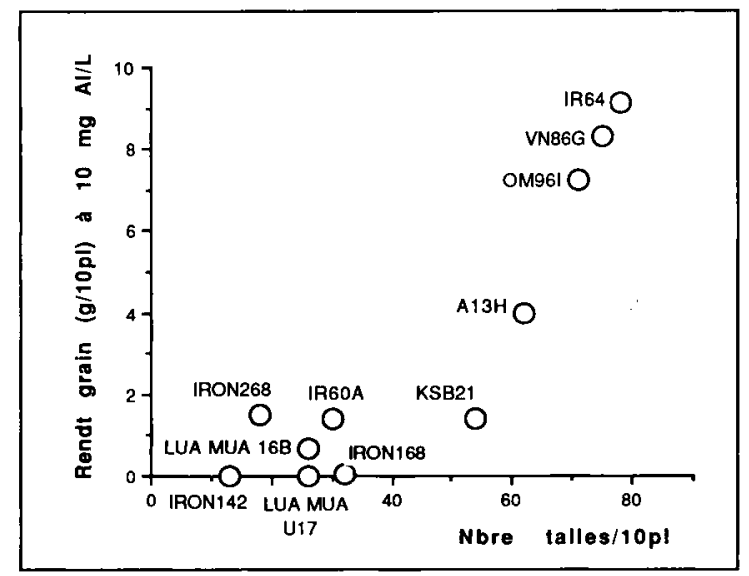

Fig 3. Relation entre la production de grains fertiles à $10 \mathrm{mg}$ Al. $L^{-1}$ et le nombre total de talles. que en effet que le tallage des céréales est une phase particulièrement sensible aux variations des conditions du milieu, telles que la disponibilité en eau, l'intensité lumineuse, la température, la nutrition azotée, etc (Connor, 1975 ; Thorne et Wood, 1987 ; Comberato et Bock, 1990). En l'absence de stress physiologique, la production de talles n'est pas nécessairement une assurance de rendement en grain vu les relations complexes qui gèrent les équilibres entre le développement des organes végétatifs et reproductifs (Shanahan et al, 1985). Ceci est confirmé par nos données à $2 \mathrm{mg} \mathrm{Al} \cdot \mathrm{L}^{-1}$ où le coefficient de corrélation entre le nombre total de talles (fertiles ou stériles) et la production de grain n'est que de 0,18. En revanche, le maintien d'une bonne capacité de tallage sous stress pourrait s'avérer un atout important de résistance à celui-ci, comme il a été montré pour le blé sous stress hydrique (Keim et Kronstad, 1981).

Par ailleurs, aucune corrélation significative n'a été trouvée entre les rapports des valeurs des caractéristiques étudiées à 2 et $10 \mathrm{mg} \mathrm{Al} \cdot \mathrm{L}^{-1}$ et la chute relative du rendement en grain.

Pour terminer, il est utile d'examiner comment des manifestations de sensibilité à l'aluminium souvent considérées dans la littérature peuvent rendre compte d'un classement de tolérance basé sur le rendement en grains. À cet effet, on a comparé les moyennes des caractéristiques mesurées relatives à 2 classes de cultivars : une première classe (classe 1) composée des 4 cultivars les plus productifs à $10 \mathrm{mg} \mathrm{Al} \cdot \mathrm{L}^{-1}$ (IR 64, VN 86G, OM 961, A 13H), et une deuxième classe (classe 2) composée de 4 cultivars moins productifs à $10 \mathrm{mg} \mathrm{Al} \cdot \mathrm{L}^{-1}$, mais productifs à 2 mg Al $\cdot L^{-1}$ (IR 60A, LUA MUA 16B, KSB 21, IRON 168). Les résultats sont donnés au tableau II.

On remarque que le développement moyen des parties aériennes et des racines n'est pas significativement différent entre les 2 classes de cultivars. Or, ces critères ont été les plus régulièrement retenus pour classer les variétés selon leur tolérance à l'aluminium. Signalons ici qu'aucune corrélation intéressante n'a non plus été mise en évidence entre les baisses relatives de croissance aérienne et racinaire et les autres caractéristiques mesurées.

Les 2 classes ne diffèrent pas non plus par leur capacité de bloquer l'aluminium au niveau racinaire et de limiter sa translocation vers les parties aériennes, alors qu'il a été quelquefois 
Tableau II. Comparaison des caractéristiques moyennes de 4 cultivars tolérants (classe 1) et de 4 cultivars sensibles (classe 2) à l'aluminium, en présence de 2 et $10 \mathrm{mg} \mathrm{Al \cdot L-1}$.

\begin{tabular}{|c|c|c|c|c|c|c|c|c|c|c|}
\hline & \multicolumn{5}{|c|}{$2 m g A l \cdot L^{-1}$} & \multicolumn{5}{|c|}{$10 \mathrm{mg} \mathrm{Al} \cdot \mathrm{L}^{-1}$} \\
\hline & \multicolumn{2}{|c|}{ Classe 1} & \multicolumn{2}{|c|}{ Classe 2} & \multirow{2}{*}{$\frac{\text { Signif a }}{\text { NS }}$} & \multicolumn{2}{|c|}{ Classe 1} & \multicolumn{2}{|c|}{ Classe 2} & \multirow{2}{*}{$\begin{array}{c}\text { Signif a } \\
{ }_{\star \star}\end{array}$} \\
\hline Grains (g/10 pl) & $24,8($ & $(5,1)^{\mathrm{b}}$ & 27,6 & $(9,5)$ & & 7,15 & $(2,25)$ & 0,90 & $(0,66)$ & \\
\hline \multicolumn{11}{|c|}{ Poids sec à $35 j(g / 15 p l)$} \\
\hline Feuilles & 1,36 & $(0,25)$ & 1,50 & $(0,32)$ & NS & 1,44 & $(0,17)$ & 1,48 & $(0,29)$ & NS \\
\hline Racines & 0,37 & $(0,08)$ & 0,44 & $(0,18)$ & NS & 0,30 & $(0,03)$ & 0,28 & $(0,09)$ & NS \\
\hline \multicolumn{11}{|c|}{ Teneurs à 35 j (mg/g MS) } \\
\hline Al feuilles & 0,03 & $(0,01)$ & 0,02 & $(0,01)$ & NS & 0,17 & $(0,02)$ & 0,16 & $(0,05)$ & NS \\
\hline Al racines & 0,52 & $(0,09)$ & 0,50 & $(0,15)$ & NS & 2,17 & $(0,12)$ & 2,17 & $(0,05)$ & NS \\
\hline Ca feuilles & 0,66 & $(0,03)$ & 0,56 & $(0,11)$ & NS & 0,95 & $(0,14)$ & 1,00 & $(0,31)$ & NS \\
\hline Ca racines & 0,82 & $(0,37)$ & 0,55 & $(0,60)$ & NS & 1,91 & $(0,42)$ & 2,42 & $(0,74)$ & NS \\
\hline Mg feuilles & 5,78 & $(2,09)$ & 5,39 & $(2,19)$ & NS & 3,18 & $(1,89)$ & 2,90 & $(0,15)$ & NS \\
\hline Mg racines & 1,87 & $(0,43)$ & 1,80 & $(0,43)$ & NS & 2,50 & $(1,02)$ & 1,99 & $(0,22)$ & NS \\
\hline$P$ feuilles & 3,54 & $(0,63)$ & 4,32 & $(1,38)$ & NS & 2,72 & $(1,02)$ & 1,80 & $(0,72)$ & NS \\
\hline P racines & 1,51 & $(0,20)$ & 1,46 & $(0,04)$ & NS & 4,56 & $(0,44)$ & 4,03 & $(0,97)$ & NS \\
\hline $\mathrm{K}$ feuilles & 21,1 & $(2,3)$ & 20,0 & $(1,7)$ & NS & 24,4 & $(0,8)$ & 23,6 & $(1,2)$ & NS \\
\hline $\mathrm{K}$ racines & 15,2 & $(1,3)$ & 16,2 & $(2,2)$ & NS & 14,6 & $(0,7)$ & 12,9 & $(2,3)$ & NS \\
\hline Nbre talles/10pl & 75,0 & $(16,9)$ & 58,3 & $(20,8)$ & NS & 71,5 & $(7,0)$ & 35,5 & $(12,6)$ & $\star \star$ \\
\hline
\end{tabular}

a Niveaux de signification des différences entre moyennes : NS : non significatif, ${ }^{* *}$ : hautement significatif $(\alpha 0.01) ;{ }^{b}$ Entre parenthèses : écarts-types.

suggéré qu'une telle propriété peut atténuer la toxicité aluminique. Il n'apparaît pas non plus que les variétés plus tolérantes gardent, en présence d'aluminium, une meilleure capacité de translocation du magnésium. En ce qui concerne la translocation du phosphore, les variétés plus tolérantes paraissent moins affectées que les variétés sensibles. En effet, la concentration moyenne en $\mathrm{P}$ dans les parties aériennes chute

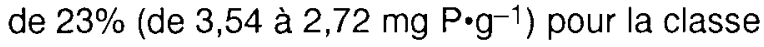

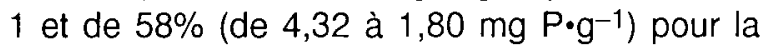
classe 2. Ceci confirme la sensiblité de la nutrition phosphatée à l'aluminium. L'absence de signification statistique ne permet cependant pas de faire de cette caractéristique un critère précoce fiable de criblage variétal par rapport à la toxicité aluminique.

Au niveau du tallage, les différences entre les 2 classes apparaissent très hautement significatives à $10 \mathrm{mg} \mathrm{Al} \mathrm{L}^{-1}$, comme on pouvait le dé- duire de la figure 3. Mais il apparaît aussi que les cultivars les plus tolérants à l'aluminium (classe 1) ont, en moyenne, une capacité intrinsèque de tallage supérieure à celle des autres cultivars en l'absence de stress aluminique. Mais, à nouveau, comme pour la nutrition phosphatée, le niveau de signification statistique est insuffisant, de sorte que la présente étude ne peut pas avancer de critère prédictif de sensibilité à l'aluminium sur la base de caractéristiques intrinsèques en l'absence de stress aluminique.

On peut enfin se demander si le nombre inférieur de talles des cultivars de la classe 2 sous stress aluminique explique totalement la moindre production de grain. C'est de fait le cas puisque la proportion de talles fertiles n'était pas significativement différente pour les 2 classes $(30 \%$ et $27 \%$ respectivement pour les classes 1 et 2 à 10 $\mathrm{mg} \mathrm{Al} \cdot \mathrm{L}^{-1}$, ces proportions étant de 68 et $66 \%$ à $2 \mathrm{mg} \mathrm{Al} \cdot \mathrm{L}^{-1}$ ) ; le nombre moyen de grains pleins 
par panicule et le poids moyen de 1000 grains n'étaient pas non plus significativement différents entre les 2 classes.

\section{CONCLUSIONS}

Cette note pose le problème de la pertinence de critères précoces de résistance à la toxicité aluminique chez le riz. L'examen de la validité de tels critères ne peut se faire que sur la base de la connaissance des rendements en grain. Même si la poursuite de cultures sur solutions nutritives jusqu'à maturité est longue, fastidieuse, voire criticable par rapport à des conditions climatiques de plein champ, on ne voit pas comment échapper à cette démarche, si l'on veut isoler les effets propres à l'aluminium, en faisant abstraction des effets parallèles inévitables observés in situ en sols acides. Cette étude met, à tout le moins, en évidence que l'établissement de classes de tolérance à l'aluminium sur la base du développement végétatif est une opération hasardeuse pour des plantes cultivées en vue de la production de grain.

Il apparaît aussi que les domaines de concentration en $\mathrm{Al}$ fréquemment utilisés dans des études de ce genre sont trop étendus. En effet, le maintien d'un développement végétatif important en présence de concentrations élevées en Al ne signifie pas qu'un rendement en grain appréciable puisse encore être escompté dans ces conditions. Dans la présente étude, le rendement moyen de 11 cultivars a chuté de $85 \%$ pour des concentration en $\mathrm{Al}$ passant de 2 à $10 \mathrm{mg} \cdot \mathrm{L}^{-1}$, alors qu'aucune réduction des parties aériennes n'était observée après 35 j et que le poids de racines avait diminué de $45 \%$.

Certaines hypothèses souvent avancées pour expliquer les différences de tolérance à l'aluminium n'ont pas été confirmées dans cette étude. II en est ainsi de la capacité différentielle des espèces ou variétés de bloquer l'aluminium au niveau racinaire et de limiter sa translocation. II en est ainsi de la capacité différentielle des cultivars d'assurer la translocation du magnésium en présence d'aluminium. II apparaît en revanche, mais sans assise statistique ferme, que la nutrition phosphatée est moins affectée chez les cultivars tolérants que chez les cultivars sensibles à l'aluminim.

Cette étude débouche cependant sur une voie de recherche prometteuse. En effet, le maintien de la capacité de tallage en présence d'alumi- nium s'est avéré être un critère très sensible pour le classement des cultivars par rapport à leur production de grain sous stress aluminique. La capacité de tallage n'était pas significativement affectée par l'aluminum pour la classe de variétés résistantes, alors que cette capacité était réduite en moyenne de $40 \%$ pour la classe des variétés sensibles. La fertilité de ces talles était fortement diminuée en présence d'aluminium, mais elle restait similaire pour les 2 classes, de sorte qu'il ne devrait pas être nécessaire d'attendre l'épiaison pour juger de la tolérance des cultivars à l'aluminium.

\section{REMERCIEMENTS}

Les auteurs remercient la Commission des Communautés européennes (DG XII) pour le soutien financier accordée à cette recherche. Celle-si s'intégrait dans le projet TS2-0257-B sur la mise en valeur des sols acides du delta du Mékong.

\section{RÉFÉRENCES}

Barnhisel R, Bertsch PM (1982) Aluminium. In: Methods of soil analysis. Part 2 (AL Page, ed) Amer Soc Agron, Madison, 275-300

Camberato JJ, Bock BR (1990) Spring wheat response to enhanced ammonium supply: II Tillering. Agron J 82, 467-473

Connor DJ (1975) Growth, water relations and yield in wheat. Austr J Plant Physiol 2, 353-366

Fageria NK, Baligar VC, Wright RJ (1988a) Aluminum toxicity in crop plants. J Plant Nutr 11, 309-319

Fageria NK, Wright RJ, Baligar VC (1988b) Rice cultivar response to aliminum in nutrient solution. Commun Soil Sci Plant Anal 19, 1133-1142

Jan F, Pettersson S (1989) Varietal diversity of upland rice sensitivity to aluminium. J Plant Nutr $12,973-993$

Keim DL, Kronstad WE (1981) Drought response of winter wheat cultivars grown under field stress conditions. Crop Sci 21, 11-15

Kinraide TB (1988) Proton extrusion by wheat roots exhibiting severe aluminum toxicity symptoms. Plant Physiol 88, 418-423

Lindsay WL (1979) Chemical equilibria in soils. John Wiley, New York

Olsen SR, Sommers LE (1982) Phosphorus. In: Methods of soil analysis. Part 2 (AL Page, ed) Amer Soc Agron, Madison, 403-430

Philippeau G (1986) Comment interpréter les résultats d'une analyse en composantes principales ? Inst Techn Céréales Fourrages, Paris 
Robson AD (1989) Soil acidity and plant growth. Academic Press, London

Shanahan JF, Donnelly KJ, Smith DH, Smika DE (1985) Shoot development properties associated with grain yield in winter wheat. Crop Sci 25, 770775

Sposito G (1989) The chemistry of soils. Oxford Univ Press, New York

Taylor GJ (1991) Current views of the aluminum stress response; the physiological basis of tolerance. Current Topics in Plant Biochemistry 10, 5793
Tang Van Hai, Truong Thi Nga, Laudelout H (1989) Effect of aluminium on the mineral nutrition of rice. Plant and Soil 114, 173-185

Thorne GN, Wood DW (1987) Effect of radiation and temperature on tiller survival, grain number and grain yield in winter wheat. Ann Bot 59, 413-426

Van Wambeke A (1976) Formation, distribution and consequences of acid soils in agricultural development. In: Plant adaptation to mineral stress in problem soils (MJ Wright, ed) Cornell Univ Press, Ithaca, NY, 15-24

Wright RJ (1989) Soil aluminum toxicity and plant growth. Commun Soil Sci Plant Anal 20, 1479-1497 